\title{
A UTILIZAÇÃO DO LASER DE BAIXA INTENSIDADE E ALTA INTENSIDADE NA ODONTOLOGIA: UMA REVISÃO INTEGRADA
}

\author{
THE USE OF LOW INTENSITY AND HIGH INTENSITY LASER IN \\ DENTISTRY: AN INTEGRATED REVIEW
}

Dimas Soares de Abreu Filho ${ }^{1}$

Pedro José Targino Ribeiro ${ }^{2}$

RESUMO: Introdução: O primeiro laser surgiu na década de 1960, e, desde então, vários estudos foram desenvolvidos a respeito das suas propriedades de fotobiomodulação através da sua irradiação nas células e tecidos animais. Objetivo: Esta revisão de literatura integrativa teve como objetivo esclarecer as diferenças entre os lasers de alta intensidade e os lasers de baixa intensidade, e avaliar sua utilização na odontologia, dissertando sobre os seus efeitos terapêuticos nos tecidos bucais. Com base nos achados bibliográficos atuais, busca-se entender os efeitos bioestimulantes a nível celular dos lasers de baixa potência, em virtude do seu uso como fator principal ou coadjuvante no controle e avanço dos processos inflamatórios, alívio da dor e cicatrização das feridas. Métodos: Através desta pesquisa de caráter qualitativo, espera-se comprovar a efetividade da terapia fotobiomoduladora, assim como evidenciar a aplicabilidade do laser nas principais áreas da odontologia, e, por fim, proporcionar uma ampla visão a respeito da importância de conhecer essa tecnologia com o propósito de trazer mais conforto ao paciente, agregando mais valor e referência ao cirurgião-dentista. A pesquisa foi realizada em plataformas de dados LILACS, SCIELO e PUBMED, foram selecionados estudos que comprovem a eficácia da utilização do laser de baixa intensidade e alta intensidade, nas respectivas áreas de atuação da odontologia, com publicações no período de 2016 e 2021, somente estudos com ensaio clínico serão citados na pesquisa. Resultados e discussão: Após o levantamento de 47 textos em potencial, destes, 28 foram excluídos por não apresentarem os critérios inclusão da pesquisa, restando apenas 19 artigos. Dos artigos em questão, os autores demonstram a diferença entre os lasers utilizados em suas pesquisas e avaliam sua utilização na odontologia, dissertando sobre seus efeitos terapêuticos nos tecidos bucais, é de grande importância que o profissional entenda o quanto os

1 Graduando em Odontologia da Faculdade Santa Maria - FSM.

2 Docente em Odontologia da Faculdade Santa Maria - FSM. 
lasers podem ser úteis na redução das sintomatologias dolorosas no pós-operatório de determinadas condutas odontológicas. Conclusão: Contudo, sabe se que os lasers de baixa intensidade são de grande utilidade na rotina de consultório odontológico sempre que houver a necessidade de promover analgesia, reparo tecidual, modulação da inflamação ou terapia antimicrobiana, os lasers de alta potência conseguem promover incisões cirúrgicas com total controle e livre de sangramento em alguns casos, além do seu efeito biomodulador em certo grau. É notório saber que as terapias com laser de baixa e alta intensidade, estão cada vez mais em evidência na mão do odontologista, em virtude dos seus benefícios e conforto ao paciente, durante e após os procedimentos.

Palavras chave: Laser. Terapia com Luz de Baixa Intensidade. Terapia a laser.

ABSTRACT: Introduction: The first laser appeared in the 1960s, and since then, several studies have been developed regarding its photobiomodulation properties through its irradiation in animal cells and tissues. Objective: This integrative literature review aimed to clarify the differences between high-intensity lasers and low-intensity lasers, and to evaluate their use in dentistry, disserting about your therapeutic effects on oral tissues. Based on current bibliographic findings, we seek to understand the biostimulating effects at the cellular level of low-power lasers, due to their use as a main or supporting factor in the control and advancement of inflammatory processes, pain relief and wound healing. Methods: Through this qualitative research, it is expected to prove the effectiveness of photobiomodulatory therapy, as well as to demonstrate the applicability of the laser in the main areas of dentistry, and, finally, to provide a broad vision regarding the importance of knowing this technology for the purpose to bring more comfort to the patient, adding more value and reference to the dentist. The research was carried out on LILACS, SCIELO and PUBMED data platforms, studies were selected that prove the effectiveness of the use of low-intensity and high-intensity lasers, in the respective areas of performance of dentistry, with publications in the period 2016 and 2021, only studies with clinical trials will be cited in the research. Results and discussion: After surveying 47 potential texts, 28 of these were excluded for not meeting the criteria for inclusion in the research, leaving only 19 articles. Of the articles in question, the authors demonstrate the difference between the lasers used in their research and evaluate their use in dentistry, discussing their therapeutic effects on oral tissues. It is very important that professionals understand how useful lasers can be in reducing of painful symptoms in the postoperative period of certain dental procedures. Conclusion: However, It is known that low-intensity lasers are very useful in the routine of the dental office whenever there is a need to promote analgesia, tissue repair, inflammation modulation or antimicrobial therapy, high-power lasers can promote surgical incisions with total control and freedom bleeding in some cases, in addition to its biomodulating effect to a certain degree. It is notorious to know that low and high intensity laser therapies are increasingly in evidence in the hand of dentists, due to their benefits and comfort to the patient, during and after the procedures.

Keywords: Laser. Low Intensity Light Therapy. Laser therapy. 


\section{INTRODUÇÃO}

A palavra laser refere-se a uma sigla que do inglês é denominada de "light amplification by stimulated emission of radiation", que significa "amplificação da luz por emissão estimulada de radiação" (LÍNS et al., 2010). O primeiro laser surgiu em 1960, porém, somente a partir de 1966 o primeiros estudos e pesquisas sobre o seu efeito de fotobiomodulação foi comprovado. A partir desses estudos iniciais, foi identificado que o efeito do laser depende de como a energia é conduzida ao tecido e sua dosagem, portanto, os estudos atuais têm como princípio entender a melhor forma de utilizá-los, considerando seus mecanismos de ação, e efeitos ao tecido humano. (LíNS et al., 2010).

Existem duas classificações em relação ao tipo de laser, o laser de baixa potência (LBP) e o laser de alta potência (LAP). Lasers de baixa potência apresentam uma absorção superficial ou em profundidade, dependendo do seu comprimento de onda são capazes de promover a fotobiomodulação, ou seja, diminuição do tempo de reparação tecidual, efeito de modulação na inflamação e analgesia. Lasers de alta potência são utilizados geralmente em procedimentos cirúrgicos ou de remoção, atuam pelo aumento da temperatura do local, e através da vaporização e ablação, são capazes de remover tecido mole e tecido duro, de acordo com os estudos de Garcez (2021).

Uma das principais vantagens é a fotobiomodulação feita com laser de baixa potência. Consiste na estimulação tecidual a nível celular. Assim que a radiação é absorvida pelos tecidos, ela estimula os fotorreceptores espalhados pelas organelas das células, em especial a mitocôndria, responsável pela produção de energia. Além disso, ocorre estímulo na liberação de prostaglandinas, bradicininas, serotoninas e histaminas que fazem relação com processos de dor e inflamação, estimula a ativação dos linfócitos, aumenta a atividade fagocitária dos macrófagos em nível vascular, aumenta a proliferação das células endoteliais e estimula a formação de tecido de granulação, onde ocorre aumento rápido de fibroblastos e fibras de 
colágeno. Diante dessas características, ocorre uma aceleração do reparo tecidual, o que alivia a dor e minimiza a inflamação (LínS et al., 2010).

Os lasers têm sido de grande importância na odontologia, sendo facilmente aplicados em todas as suas áreas de conhecimento, agindo como primeira escola, ou de forma coadjuvante, ao protocolo de tratamento do paciente; cada laser disponível no mercado apresenta suas determinadas potências e indicações.

O objetivo do presente estudo foi realizar uma revisão de literatura sobre a utilização do LBP e LAP nas áreas de atuação da odontologia. A finalidade do estudo foi remeter à importância do conhecimento a respeito dessa tecnologia, trazendo muito mais conforto ao paciente em procedimentos cirúrgicos, tratamento de lesões orais, pós-operatórios clínicos e no controle de infecções.

\section{METODOLOGIA}

Este estudo tem como base uma pesquisa qualitativa, de caráter integrativo. Foram selecionados estudos que tinham relevância e que eram propícios ao tema proposto.

Foi adotado o método de pesquisa bibliográfico, que é desenvolvido com base em informações adquiridas de materiais já elaborados. Foram selecionados estudos que justificassem o tema da utilização do laser de baixa intensidade e alta intensidade na odontologia, com publicação no período de 2016 a 2021, que apresentasse a relação com a aplicabilidade do laser nas respectivas áreas de atuação da odontologia. Foram selecionados estudos através das plataformas Latin American and Caribbean Healt Sciences Literature (LILACS), Scientific Electronic Library Online (SCIELO), GOOGLE SCHOLAR e National Library of Medicine National Institutes of Health dos EUA (MEDLINE/PUBMED) em idioma português e inglês, dos tipos revisão sistemática, relato de caso e ensaio clínico que estejam de acordo com os critérios de inclusão. Os resultados da pesquisa foram sintetizados e apresentados na seção de resultados. 
Foram selecionados e incluídos estudos completos, realizados com seres humanos, e que tratem da utilização do laser na odontologia (em qualquer área de atuação). Dentre os artigos disponíveis para a consulta, foram elegidos, principalmente, aqueles que apresentaram uma abordagem explanativa relacionados a estudos de protocolos cirúrgicos ou terapêuticos com laser, fotobiomodulação e terapia fotodinâmica.

Não foram selecionadas revisões da literatura, projetos em andamento ou estudos com conflitos de interesse. Não foi utilizada nenhuma pesquisa, na qual apresente, durante sua análise, distanciamento do tema referente à pesquisa em questão.

\section{RESULTADOS}

Ao todo foram reunidos cerca de 47 textos ( 1 em português e 46 em inglês), em potencial, após o levantamento bibliográfico, que estão validados entre os anos de 2016 e 2021. Destes, 28 foram excluídos por não apresentarem os critérios de inclusão da pesquisa, não disponibilizarem o texto completo ou serem repetidos, restando apenas 19 artigos.

Dentre os resultados das buscas realizadas, cerca de 100\% dos artigos (19 artigos) comprovaram a eficácia da utilização do laser de baixa intensidade ou alta intensidade na sua respectiva área da odontologia, enquanto somente $0 \%$ dos artigos (nenhum artigo) não relatam eficácia na utilização dos mesmos. 
Tabela 1: Resultados obtidos através das seguintes plataformas de dados online.

\begin{tabular}{|c|c|c|c|c|c|c|c|}
\hline \multirow{2}{*}{ Descritores } & \multicolumn{2}{|c|}{ PUBMED } & \multicolumn{2}{c|}{ SCIELO } & \multicolumn{2}{c|}{ LILACS } & \multirow{2}{*}{ Total } \\
\cline { 2 - 8 } & Inglês & Português & Inglês & Português & Inglês & Português & \\
\hline $\begin{array}{l}\text { Terapia a laser oral } \\
\text { de alto nível (high } \\
\text { level oral laser } \\
\text { therapy) e terapia a } \\
\text { laser oral de baixo } \\
\text { nível (low level oral } \\
\text { laser therapy) }\end{array}$ & 39 & 0 & 7 & 1 & 0 & 0 & 47 \\
\hline
\end{tabular}

Quadro 2. Artigos selecionados para revisão de literatura no período de 2016 2021. De acordo com os autores, com o ano da publicação, com o título do artigo, com o objetivo, com o tipo de estudo, e com a conclusão.

\begin{tabular}{|c|c|c|c|c|}
\hline $\begin{array}{l}\text { AUTOR } \\
\text {-ANO }\end{array}$ & $\begin{array}{l}\text { TIPO DE } \\
\text { ESTUDO }\end{array}$ & TíTULO & OBJETIVO & CONCLUSÃO \\
\hline $\begin{array}{l}\text { WANG } \\
\text { et al. } \\
2020\end{array}$ & $\begin{array}{l}\text { Ensaio } \\
\text { clínico }\end{array}$ & $\begin{array}{l}\text { Laser-Assisted } \\
\text { Regenerative Surgical } \\
\text { Therapy for Peri- } \\
\text { implantitis: A Randomized } \\
\text { Controlled Clinical Trial }\end{array}$ & $\begin{array}{l}\text { O laser pode auxiliar } \\
\text { na redução da bolsa } \\
\text { para peri-implantite? }\end{array}$ & $\begin{array}{l}\text { A terapia com laser } \\
\text { pode ajudar a } \\
\text { melhorar a redução da } \\
\text { profundidade da bolsa } \\
\text { de sondagem. }\end{array}$ \\
\hline $\begin{array}{l}\text { MADAN } \\
\text { l et al. } \\
2019\end{array}$ & $\begin{array}{l}\text { Ensaio } \\
\text { clínico }\end{array}$ & $\begin{array}{l}\text { A randomized clinical trial } \\
\text { comparing the efficacy of } \\
\text { low-level laser therapy } \\
(\text { LLLT) and laser } \\
\text { acupuncture therapy } \\
\text { (LAT) in patients with } \\
\text { temporomandibular } \\
\text { disorders }\end{array}$ & $\begin{array}{l}\text { Comparar a eficácia } \\
\text { da terapia a laser de } \\
\text { baixa intensidade } \\
\text { (LLLT) com a terapia } \\
\text { de acupuntura a } \\
\text { laser (LAT) em } \\
\text { pacientes rom } \\
\text { disfunções } \\
\text { temporomandibulare } \\
\text { s (DTMs). }\end{array}$ & $\begin{array}{l}\text { Tanto o LLLT quanto o } \\
\text { LAT foram eficazes na } \\
\text { redução da dor e no } \\
\text { aumento do } \\
\text { movimento mandibular } \\
\text { excursivo e protrusivo } \\
\text { em pacientes com } \\
\text { DTM. O LAT pode ser } \\
\text { sugerido como uma } \\
\text { alternativa adequada } \\
\text { a LLLT, pois fornece } \\
\text { resultados eficazes e } \\
\text { ocupa menos tempo } \\
\text { na cadeira. }\end{array}$ \\
\hline $\begin{array}{l}\text { OKAMO } \\
\text { TO et al. } \\
2020\end{array}$ & $\begin{array}{l}\text { Ensaio } \\
\text { clínico }\end{array}$ & $\begin{array}{lll}\text { Photodynamic therapy for } \\
\text { endodontic } & \text { treatment } & \text { of } \\
\text { primary } & \text { teeth: } & \text { A } \\
\text { randomized } & \text { controlled } \\
\text { clinical trial } & & \end{array}$ & 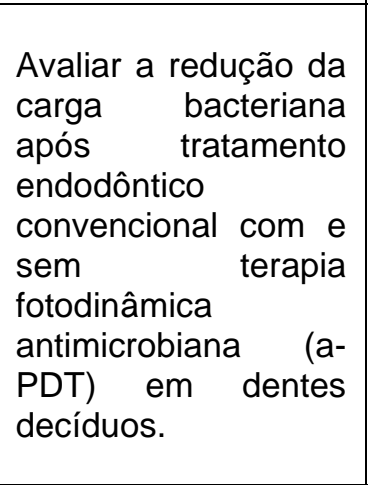 & $\begin{array}{l}\text { O tratamento } \\
\text { convencional } \\
\text { combinado com a TFD } \\
\text { antimicrobiana com os } \\
\text { parâmetros usados } \\
\text { neste estudo se } \\
\text { mostrou eficaz, mas } \\
\text { apresentou } \\
\text { capacidade eficaz } \\
\text { igual ao tratamento } \\
\text { endodôntico } \\
\text { convencional sozinho. }\end{array}$ \\
\hline $\begin{array}{l}\text { WU et } \\
\text { al. } 2018\end{array}$ & $\begin{array}{l}\text { Ensaio } \\
\text { clínico }\end{array}$ & $\begin{array}{l}\text { Effect of low-level laser } \\
\text { therapy on tooth-related }\end{array}$ & $\begin{array}{l}\text { Avaliar o efeito da } \\
\text { LLLT na dor e na }\end{array}$ & $\begin{array}{ll}\text { Uma } & \text { aplicação } \\
\text { repetida de LLLT foi }\end{array}$ \\
\hline
\end{tabular}




\begin{tabular}{|c|c|c|c|c|}
\hline & & $\begin{array}{l}\text { pain and somatosensory } \\
\text { function evoked by } \\
\text { orthodontic treatment }\end{array}$ & $\begin{array}{l}\text { sensibilização } \\
\text { somatossensorial } \\
\text { induzida pelo } \\
\text { tratamento } \\
\text { ortodôntico. }\end{array}$ & $\begin{array}{l}\text { capaz de reduzir } \\
\text { significativamente os } \\
\text { escores de dor } \\
\text { autorreferidos e a } \\
\text { sensibilização dos } \\
\text { tecidos periodontal e } \\
\text { gengival evocados } \\
\text { pelo tratamento } \\
\text { ortodôntico. }\end{array}$ \\
\hline $\begin{array}{l}\text { ALSAY } \\
\text { ED } \\
\text { HASAN } \\
(2016)\end{array}$ & $\begin{array}{l}\text { Ensaio } \\
\text { clínico }\end{array}$ & $\begin{array}{l}\text { Low-level laser therapy } \\
\text { effectiveness }\end{array} \begin{array}{l}\text { in } \\
\text { accelerating orthodontic } \\
\text { tooth movement: A } \\
\text { randomized controlled } \\
\text { clinical trial }\end{array}$ & $\begin{array}{l}\text { Avaliar a eficácia da } \\
\text { terapia a laser de } \\
\text { baixa intensidade } \\
\text { (LLLT) na aceleração } \\
\text { da movimentação } \\
\text { dentária ortodôntica } \\
\text { de } r \text { incisivos } \\
\text { superiores } \\
\text { apinhados. }\end{array}$ & $\begin{array}{l}\text { A terapia a laser de } \\
\text { baixa intensidade, } \\
\text { utilizada com os } \\
\text { parâmetros descritos, } \\
\text { é um método eficaz } \\
\text { para acelerar a } \\
\text { movimentação } \\
\text { dentária ortodôntica } \\
\text { em casos de } \\
\text { apinhamento dentário. }\end{array}$ \\
\hline $\begin{array}{l}\text { ALVES } \\
\text { et al. } \\
2019\end{array}$ & $\begin{array}{l}\text { Ensaio } \\
\text { clínico }\end{array}$ & $\begin{array}{l}\text { Influence of antimicrobial } \\
\text { photodynamic } \\
\text { carious rapy in } \\
\text { Randomized split-mouth } \\
\text { clinical trial in primary } \\
\text { molars }\end{array}$ & $\begin{array}{l}\text { Avaliar o efeito da } \\
\text { terapia } \\
\text { antimicrobiana } \\
\text { fotodinâmica (aPDT) } \\
\text { na redução } \\
\text { deStreptococcus } \\
\text { mutans e seu efeito } \\
\text { nas restaurações } \\
\text { realizadas. }\end{array}$ & $\begin{array}{l}\text { O aPDT pode ser } \\
\text { usado como um } \\
\text { tratamento adicional } \\
\text { contra microrganismos } \\
\text { cariogênicos após a } \\
\text { remoção seletiva de } \\
\text { cárie sem } \\
\text { comprometer as as a } \\
\text { restaurações de resina } \\
\text { composta. }\end{array}$ \\
\hline $\begin{array}{l}\mathrm{BROCH} \\
\mathrm{ADO} \text { et } \\
\text { al. } 2019\end{array}$ & $\begin{array}{l}\text { Ensaio } \\
\text { clínico }\end{array}$ & $\begin{array}{l}\text { Comparative } \\
\text { effectiveness } \\
\text { photobiomodulation and } \\
\text { manual therapy alone or } \\
\text { combined in TMD } \\
\text { patients: a randomized } \\
\text { clinical trial }\end{array}$ & $\begin{array}{l}\text { Avaliar a eficácia da } \\
\text { fotobiomodulação } \\
\text { (BPM) associada a } \\
\text { terapia manual (MT), } \\
\text { comparando com a } \\
\text { terapia manual } \\
\text { sozinha. }\end{array}$ & $\begin{array}{l}\text { Todos os protocolos } \\
\text { testados foram } \\
\text { capazes de diminuir a } \\
\text { dor e melhorar os } \\
\text { movimentos } \\
\text { mandibulares funções } \\
\text { algumas } \\
\text { mandibulares. } \\
\text { Contudo, a associação } \\
\text { da BPM e MT não foi } \\
\text { diferente de realiza-las } \\
\text { isoladamente. }\end{array}$ \\
\hline $\begin{array}{l}\text { COELH } \\
\text { O } 2019\end{array}$ & $\begin{array}{l}\text { Ensaio } \\
\text { clínico }\end{array}$ & $\begin{array}{l}\text { The effects of } \\
\text { photodynamic therapy on } \\
\text { postoperative pain in } \\
\text { teeth with necrotic pulps }\end{array}$ & \begin{tabular}{lr}
\multicolumn{2}{l}{ Determinar os efeitos } \\
da & terapia \\
fotodinâmica & (TFD) \\
na dor & pós- \\
operatória & após \\
tratamentos & de \\
dentes com & polpas \\
necróticas. &
\end{tabular} & $\begin{array}{lr}\text { A PDT teve um efeito } \\
\text { significativo }\end{array}$ \\
\hline $\begin{array}{l}\text { DEMIR } \\
\text { KOL et } \\
\text { al. } 2017\end{array}$ & $\begin{array}{l}\text { Ensaio } \\
\text { clínico }\end{array}$ & $\begin{array}{lr}\text { Efficacy of } & \text { Low-Level } \\
\text { Laser } & \text { Therapy in } \\
\text { Subjective } & \text { Tinnitus } \\
\text { Patients randibular with } \\
\text { Temporomandibur } \\
\text { Disorders } \\
\end{array}$ & $\begin{array}{l}\text { O objetivo do } \\
\text { tratamento é eliminar } \\
\text { o zumbido ou, pelo } \\
\text { menos, diminuir seu } \\
\text { volume aparente, } \\
\text { através da terapia }\end{array}$ & $\begin{array}{l}\text { Ambos os lasers de } \\
\text { diodo Nd: YAG e } 810 \\
\text { nm foram eficazes no } \\
\text { tratamento do zumbido } \\
\text { subjetivo relacionado } \\
\text { às DTMs. }\end{array}$ \\
\hline
\end{tabular}




\begin{tabular}{|c|c|c|c|c|}
\hline & & & com LBP & \\
\hline $\begin{array}{l}\text { FIORAV } \\
\text { ANTI et } \\
\text { al. } 2021\end{array}$ & $\begin{array}{l}\text { Ensaio } \\
\text { clínico }\end{array}$ & $\begin{array}{l}\text { The Efficacy of Lingual } \\
\text { Laser Frenectomy in } \\
\text { Pediatric OSAS: A } \\
\text { Randomized } \\
\text { Blinded and } \\
\text { Clinical Study }\end{array}$ & $\begin{array}{lr}\text { Investiga como uma } \\
\text { frenectomia lingual } \\
\text { com laser de diodo } \\
\text { pode melhorar a } \\
\text { síndrome da apnéia } \\
\text { obstrutiva do rono } \\
\text { (SAOS) } & \text { em } \\
\text { pacientes } & \\
\text { pediátricos. } & \\
\end{array}$ & $\begin{array}{l}\text { Este estudo } \\
\text { demonstrou que a } \\
\text { terapia de frenectomia } \\
\text { lingual com laser de } \\
\text { diodo pode melhorar a } \\
\text { gravidade da SAOS } \\
\text { em pacientes } \\
\text { pediátricos. }\end{array}$ \\
\hline $\begin{array}{l}\text { GANDH } \\
\text { l et al. } \\
2019\end{array}$ & $\begin{array}{l}\text { Ensaio } \\
\text { clínico }\end{array}$ & $\begin{array}{l}\text { Effectiveness of } \\
\text { Adjunctive Use of Low- } \\
\text { Level Laser Therapy and } \\
\text { Photodynamic Therapy } \\
\text { After Scaling and Root } \\
\text { Planing in Patients with } \\
\text { Chronic Periodontitis }\end{array}$ & $\begin{array}{lr}\text { Avaliar a eficácia da } \\
\text { terapia a laser de } \\
\text { baixa intensidade } \\
(\text { LLLT) e da } & \text { terapia } \\
\text { fotodinâmica } & \text { (PDT) } \\
\text { como } & \text { um } \\
\text { complemento } & \text { para } \\
\text { raspagem } & \text { e } \\
\text { alisamento radicular } \\
\text { (SRP) no tratamento } \\
\text { da } & \text { periodontite } \\
\text { crônica } & \\
\end{array}$ & $\begin{array}{l}\text { A terapia periodontal } \\
\text { não cirúrgica de } \\
\text { periodontite crônica } \\
\text { usando PDT e LLLT } \\
\text { como um adjunto ao } \\
\text { SRP foi } \\
\text { significativamente } \\
\text { mais eficaz do que } \\
\text { SRP sozinho. Durante } \\
\text { as visitas de } \\
\text { acompanhamento. }\end{array}$ \\
\hline $\begin{array}{l}\text { LOPES } \\
\text { et al. } \\
2018\end{array}$ & $\begin{array}{l}\text { Ensaio } \\
\text { clínico }\end{array}$ & $\begin{array}{l}\text { Effect } \\
\text { photobiomodulation of } \\
\text { therapy on postoperative } \\
\text { pain after endodontic } \\
\text { treatment: a randomized, } \\
\text { controlled, clinical study }\end{array}$ & 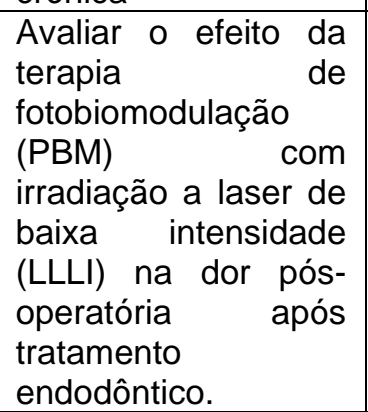 & $\begin{array}{l}\text { O efeito da terapia } \\
\text { com PBM após o } \\
\text { tratamento } \\
\text { endodôntico mostrou } \\
\text { uma diminuição } \\
\text { significativa na } \\
\text { prevalência de dor } \\
\text { pós-operatória. }\end{array}$ \\
\hline $\begin{array}{l}\text { MATAR } \\
\text { ESE et } \\
\text { al. } 2017\end{array}$ & $\begin{array}{l}\text { Ensaio } \\
\text { clínico }\end{array}$ & $\begin{array}{l}\text { The Effects of Diode } \\
\text { Laser Therapy as an } \\
\text { Adjunct to Scaling and } \\
\text { Root Planing in the } \\
\text { Treatment of Aggressive } \\
\text { Periodontitis: A } 1 \text {-Year } \\
\text { Randomized Controlled } \\
\text { Clinical Trial }\end{array}$ & $\begin{array}{l}\text { Investigar } r \text { e } \\
\text { comparar os efeitos } \\
\text { clínicos, microbianos } \\
\text { e inflamatórios de } \\
\text { um laser de diodo } \\
\text { como um adjunto } \\
\text { para raspagem e e } \\
\text { alisamento radicular } \\
\text { (SRP) versus SRP } \\
\text { sozinho para } \quad \text { o } \\
\text { tratamento } \\
\text { periodontite } \\
\text { agressiva } \\
\text { generalizada } \\
\text { (GAgP). }\end{array}$ & $\begin{array}{l}\text { Em } 1 \text { ano, o laser de } \\
\text { diodo mais SRP } \\
\text { produziu uma redução } \\
\text { significativa em alguns } \\
\text { parâmetros clínicos, } \\
\text { enquanto r as } \\
\text { alterações microbianas } \\
\text { e do mediador } \\
\text { inflamatório não foram } \\
\text { significativamente } \\
\text { reduzidas em } \\
\text { comparação com o } \\
\text { SRP sozinho. }\end{array}$ \\
\hline $\begin{array}{l}\text { METIN } \\
2018\end{array}$ & $\begin{array}{l}\text { Ensaio } \\
\text { clínico }\end{array}$ & $\begin{array}{l}\text { Effects of low-level laser } \\
\text { therapy on soft and hard } \\
\text { tissue healing after } \\
\text { endodontic surgery }\end{array}$ & $\begin{array}{lr}\text { Examinar } & \text { os } \\
\text { possíveis } & \text { benefícios } \\
\text { da terapia a laser de } \\
\text { baixa intensidade } \\
(\text { LLLT) } & \text { na } \\
\text { cicatrização } & \text { de } \\
\text { tecidos moles } \text { e } \\
\text { duros após cirurgia } \\
\text { endodôntica. }\end{array}$ & $\begin{array}{l}\text { A LLLT melhorou a } \\
\text { cicatrização dos } \\
\text { tecidos moles e duros } \\
\text { após a cirurgia } \\
\text { endodôntica e também } \\
\text { mostrou efeitos } \\
\text { favoráveis na dor e na } \\
\text { qualidade de vida dos } \\
\text { pacientes, } \\
\text { especialmente na fase } \\
\text { inicial do período de }\end{array}$ \\
\hline
\end{tabular}




\begin{tabular}{|c|c|c|c|c|}
\hline & & & & cicatrização. \\
\hline $\begin{array}{l}\text { MOHAJ } \\
\text { ERANI } \\
\text { et al. } \\
2020\end{array}$ & $\begin{array}{l}\text { Ensaio } \\
\text { clínico }\end{array}$ & $\begin{array}{l}\text { Does Combined Low- } \\
\text { Level Laser and Light- } \\
\text { Emitting Diode Light } \\
\text { Irradiation Reduce Pain, } \\
\text { Swelling, and Trismus } \\
\text { After Surgical Extraction } \\
\text { of Mandibular Third } \\
\text { Molars? A Randomized } \\
\text { Double-Blinded Crossover } \\
\text { Study }\end{array}$ & 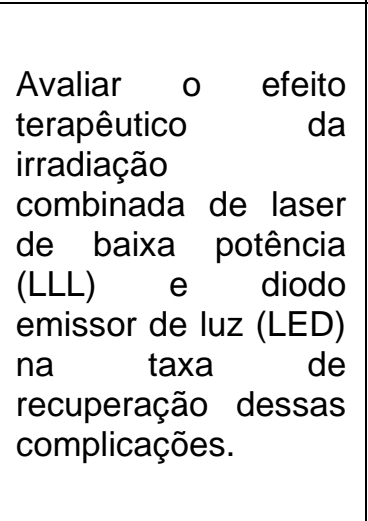 & $\begin{array}{l}\text { Os níveis de dor pós- } \\
\text { operatória nos dias } 3 \text { e } \\
7 \text { foram } \\
\text { significativamente } \\
\text { menores no grupo de } \\
\text { intervenção. Em } \\
\text { comparação com o } \\
\text { grupo de controle, o } \\
\text { nível de trismo pós- } \\
\text { operatório do grupo de } \\
\text { intervenção } \\
\text { significativamente foi } \\
\text { menor no dia 3. }\end{array}$ \\
\hline $\begin{array}{l}\text { ASUTA } \\
\text { Y et al. } \\
2021\end{array}$ & $\begin{array}{l}\text { Ensaio } \\
\text { clínico }\end{array}$ & $\begin{array}{l}\text { Three-Dimensional } \\
\text { Evaluation of the Effect of } \\
\text { Low-Level Laser Therapy } \\
\text { on Facial Swelling after } \\
\text { Lower Third Molar } \\
\text { Surgery: A Randomized, } \\
\text { Placebo-Controlled Study }\end{array}$ & 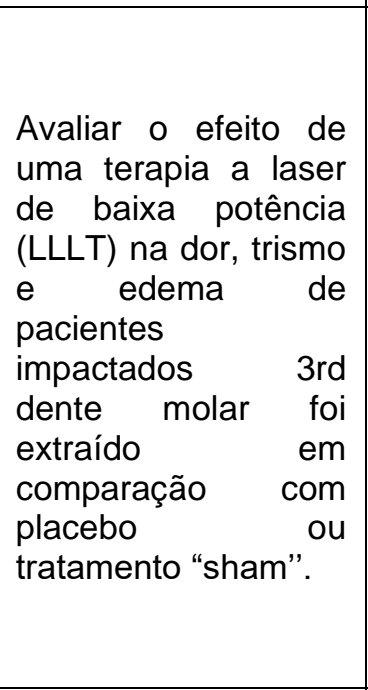 & $\begin{array}{l}\text { Foi observado que a } \\
\text { LLLT em dose única } \\
\text { reduziu a intensidade } \\
\text { da dor e o inchaço } \\
\text { facial após a cirurgia } \\
\text { do terceiro molar, } \\
\text { porém não há } \\
\text { evidências suficientes } \\
\text { para considerar a } \\
\text { terapia LLLT um } \\
\text { tratamento útil e } \\
\text { eficiente para a } \\
\text { redução das } \\
\text { morbidades pós- } \\
\text { operatórias de cirurgia } \\
\text { de terceiros molares } \\
\text { mandibulares. }\end{array}$ \\
\hline $\begin{array}{l}\text { TARAS } \\
\text { ENKO } \\
\text { et al. } \\
2021\end{array}$ & $\begin{array}{l}\text { Ensaio } \\
\text { clínico }\end{array}$ & $\begin{array}{l}\text { High-level laser therapy } \\
\text { versus scalpel surgery in } \\
\text { the treatment of oral } \\
\text { lichen planus: a } \\
\text { randomized control trial }\end{array}$ & $\begin{array}{l}\text { o Comparar a } \\
\text { eficácia clínica de } \\
\text { vários tipos de } \\
\text { terapia a laser de } \\
\text { alto nível (HLLT) em } \\
\text { relação à excisão } \\
\text { com bisturi para o } \\
\text { tratamento cirúrgico } \\
\text { do líquen plano oral } \\
\text { erosivo (LPO). }\end{array}$ & $\begin{array}{l}\text { O achado do presente } \\
\text { estudo indica que a } \\
\text { utilização do HLLT é } \\
\text { benéfica à incisão } \\
\text { tradicional de bisturi } \\
\text { para o tratamento } \\
\text { cirúrgico da forma } \\
\text { erosiva do LPO e } \\
\text { propõe o Er: YAG } \\
\text { como o HLLT mais } \\
\text { eficaz ao final do } \\
\text { primeiro mês pós- } \\
\text { operatório } \\
\text { aqueles usados neste } \\
\text { estudo. }\end{array}$ \\
\hline $\begin{array}{l}\text { URAZ et } \\
\text { al. } 2018\end{array}$ & $\begin{array}{l}\text { Ensaio } \\
\text { clínico }\end{array}$ & $\begin{array}{l}\text { Patient perceptions and } \\
\text { clinical efficacy of labial } \\
\text { frenectomies using diode } \\
\text { laser versus conventional } \\
\text { techniques }\end{array}$ & 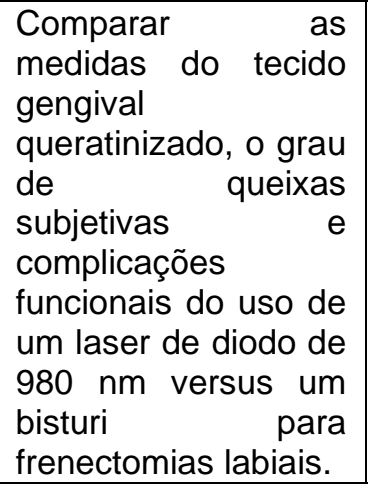 & 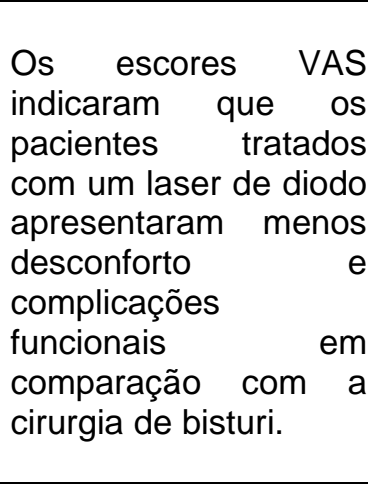 \\
\hline
\end{tabular}




\begin{tabular}{|c|c|c|c|c|}
\hline $\begin{array}{l}\text { ZHENG } \\
2021\end{array}$ & $\begin{array}{l}\text { Ensaio } \\
\text { clínico }\end{array}$ & $\begin{array}{l}\text { Clinical research: low- } \\
\text { level laser therapy } \\
\text { in accelerating orthodontic } \\
\text { tooth movement }\end{array}$ & 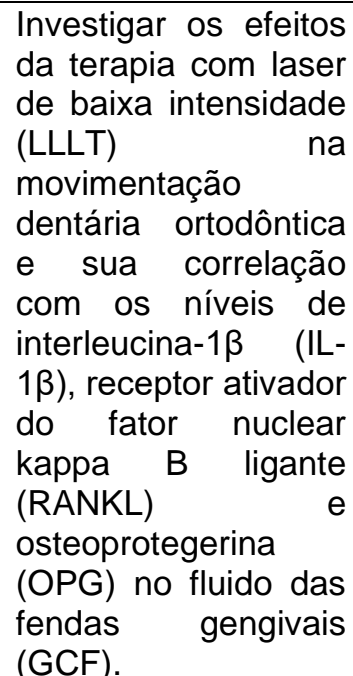 & $\begin{array}{l}\text { Com as configurações } \\
\text { dos parâmetros } \\
\text { usados neste estudo, } \\
\text { o LLLT poderia, até } \\
\text { certo ponto, levar a } \\
\text { alterações no } \\
\text { metabolismo ósseo, o } \\
\text { que poderia acelerar a } \\
\text { movimentação } \\
\text { dentária ortodôntica. }\end{array}$ \\
\hline
\end{tabular}

Levando em consideração algumas limitações encontradas ao longo desse estudo, ainda existe uma grande escassez de artigos voltados a relatar casos onde haja a utilização dos lasers, principalmente o LAP. Porém, esse estudo contribui para evidenciar a grande abrangência de procedimentos e casos onde as terapias com LAP e LBP podem atuar de forma benéfica, prática e eficiente, trazendo, assim, um maior conforto aos pacientes nas demandas odontológicas.

\section{CONCLUSÃO}

Diante dos artigos encontrados, notou-se que os LBPs trabalha em comprimentos de onda menores, assim conseguem promover efeitos benéficos aos tecidos, que através da sua irradiação pode promover muito mais do que apenas a reparação tecidual, biomodulação e analgesia, mas também o controle microbiano através da terapia fotodinâmica, que é de grande importância no combate de grande parte das patologias odontogênicas. Enquanto que os LAPs atuam em comprimento de onda maiores e dessa forma conseguem promover incisão em tecidos moles e duros através dos princípios de vaporização e ablação, além disso, conseguem promover modulação da inflamação e analgesia em pequena escala, comparado ao LBP. 


\section{REFERÊNCIAS BIBLIOGRÁFICAS}

ALSAYED H.; KINDA S.; OMAR H. "Low-level laser therapy effectiveness in accelerating orthodontic tooth movement: A randomized controlled clinical trial." The Angle orthodontist vol. 87,4 , p 1-6, (2017).

ALVES, L. V. G. L. et al. "Influence of antimicrobial photodynamic therapy in carious lesion. Randomized split-mouth clinical trial in primary molars." Photodiagnosis and photodynamic therapy vol. 26, p. 1-7, (2019).

ASUTAY, F. et al. "Three-dimensional evaluation of the effect of low-level laser therapy on facial swelling after lower third molar surgery: A randomized, placebo-controlled study." Nigerian journal of clinical practice vol. 21,9, p. 1-5, (2018).

BROCHADO, F. T. et al. "Comparative effectiveness of photobiomodulation and manual therapy alone or combined in TMD patients: a randomized clinical trial." Brazilian oral research vol. $32 \mathrm{e}$ 50 , p. 1-6, (2018).

CAVALCANTI, T. M. et al. Conhecimento das propriedades físicas e da interação do laser com os tecidos biológicos na odontologia. Anais Brasileiros de Dermatologia, v. 86, n. 5, p. 955960, (2011).

COELHO, M. S.; VILAS-BOAS, L.; TAWIL, P. Z. "The effects of photodynamic therapy on postoperative pain in teeth with necrotic pulps." Photodiagnosis and photodynamic therapy vol. 27, p. 1-3, (2019).

DEMIRKOL, N. et al. "Efficacy of Low-Level Laser Therapy in Subjective Tinnitus Patients with Temporomandibular Disorders." Photomedicine and laser surgery vol. 35,8, p. 1-4, (2017).

FIORAVANTI, M. et al. "The Efficacy of Lingual Laser Frenectomy in Pediatric OSAS: A Randomized Double-Blinded and Controlled Clinical Study." International journal of environmental research and public health vol. 18,11 6112, p 1-11, (2021).

GANDHI, K. K. et al. "Effectiveness of Adjunctive Use of Low-Level Laser Therapy and Photodynamic Therapy After Scaling and Root Planing in Patients with Chronic Periodontitis." The International journal of periodontics \& restorative dentistry vol. 39,6, p. 2-10, (2019).

GARCEZ, A. S.; NUNEZ, S. C.; RIBEIRO, M. S. Aplicação clínica do laser na odontologia. São Paulo, Brasil: Editora Manole, 2020. 9786555764406. Disponível em: https://integrada.minhabiblioteca.com.br/\#/books/9786555764406/. Acesso em: 02 dez. (2021). p.1-79.

LINS, R. D. A. U. et al. Efeitos bioestimulantes do laser de baixa potência no processo de reparo. Anais Brasileiros de Dermatologia, v. 85, n. 6, p. 849-855, (2010).

LOPES, L. P. B. et al. "Effect of photobiomodulation therapy on postoperative pain after endodontic treatment: a randomized, controlled, clinical study." Clinical oral investigations vol. 23,1, p.1-6, (2019).

MADANI, A. et al. "A randomized clinical trial comparing the efficacy of low-level laser therapy (LLLT) and laser acupuncture therapy (LAT) in patients with temporomandibular disorders." Lasers in medical Science vol. 35,1, p. 1-8, (2020).

MATARESE, G. et al. "The Effects of Diode Laser Therapy as an Adjunct to Scaling and Root 
Planing in the Treatment of Aggressive Periodontitis: A 1-Year Randomized Controlled Clinical Trial." Photomedicine and laser surgery vol. 35,12, p. 1-6, (2017).

METIN, R.; TATLI, U.; EVLICE, B. "Effects of low-level laser therapy on soft and hard tissue healing after endodontic surgery." Lasers in medical science vol. 33,8, p.1-6, (2018).

MOHAJERANI, H. et al. "Does Combined Low-Level Laser and Light-Emitting Diode Light Irradiation Reduce Pain, Swelling, and Trismus After Surgical Extraction of Mandibular Third Molars? A Randomized Double-Blinded Crossover Study." Journal of oral and maxillofacial surgery: official journal of the American Association of Oral and Maxillofacial Surgeons vol. 79,8, p.1-5, (2021).

OKAMOTO, C. B. et al. "Photodynamic therapy for endodontic treatment of primary teeth: A randomized controlled clinical trial." Photodiagnosis and photodynamic therapy vol. 30, p. 1-3, (2020).

PROCKT, A. P.; TAKAHASHI, A.; PAGNONCELLI, R. M. Uso de terapia com laser de baixa intensidade na cirurgia bucomaxilofacial. Revista Portuguesa de Estomatologia, Medicina Dentária e Cirurgia Maxilofacial, v. 49, n. 4, p. 247-255, (2008).

ROCHA J. A. M. et al. Modulação da proliferação fibroblástica e da resposta inflamatória pela terapia a laser de baixa intensidade no processo de reparo tecidual. Anais Brasileiros de Dermatologia, v. 81, n. 2, p. 150-156, (2006).

TARASENKO, S. et al. "High-level laser therapy versus scalpel surgery in the treatment of oral lichen planus: a randomized control trial." Clinical oral investigations vol. 25,10, p. 1-8, (2021).

URAZ, A. et al. "Patient perceptions and clinical efficacy of labial frenectomies using diode laser versus conventional techniques." Journal of stomatology, oral and maxillofacial surgery vol. 119,3 , p. 1-5, (2018).

WANG, C. W. et al. "Laser-assisted regenerative surgical therapy for peri-implantitis: A randomized controlled clinical trial." Journal of periodontology vol. 92,3, p. 1-15, (2021).

WU, S. et al. "Effect of low-level laser therapy on tooth-related pain and somatosensory function evoked by orthodontic treatment." International journal of oral science vol. 10,3, p. 1-5, Jul (2018).

ZHENG, J.; YANG, K. "Clinical research: low-level laser therapy in accelerating orthodontic tooth movement." BMC oral health vol. 21,1 324, p. 1-7, (2021). 